\title{
Lipid abnormalities in atopic skin are driven by type 2 cytokines
}

\author{
Evgeny Berdyshev, ${ }^{1}$ Elena Goleva, ${ }^{2}$ Irina Bronova, ${ }^{1}$ Nathan Dyjack, ${ }^{3}$ Cydney Rios, ${ }^{3}$ John Jung, ${ }^{2}$ \\ Patricia Taylor, ${ }^{2}$ Mingeum Jeong, ${ }^{4}$ Clifton F. Hall, ${ }^{2}$ Brittany N. Richers, ${ }^{2}$ Kathryn A. Norquest, ${ }^{2}$ \\ Tao Zheng, ${ }^{4}$ Max A. Seibold, ${ }^{2,3,5}$ and Donald Y.M. Leung ${ }^{2,6,}$ \\ 'Department of Medicine, ${ }^{2}$ Department of Pediatrics, and ${ }^{3}$ Center for Genes, Environment and Health, National Jewish \\ Health, Denver, Colorado. ${ }^{4}$ Departments of Pediatrics and Internal Medicine, Yale University, New Haven, Connecticut, \\ USA. ${ }^{5}$ Division of Pulmonary Sciences and Critical Care Medicine, Department of Medicine, University of Colorado Denver, \\ Denver, Colorado, USA. ${ }^{6}$ Department of Pediatrics, University of Colorado, Denver, Colorado, USA.
}

\begin{abstract}
Lipids in the stratum corneum of atopic dermatitis (AD) patients differ substantially in composition from healthy subjects. We hypothesized that hyperactivated type 2 immune response alters $A D$ skin lipid metabolism. We have analyzed stratum corneum lipids from nonlesional and lesional skin of AD subjects and IL-13 skin-specific Tg mice. We also directly examined the effects of IL-4/ IL-13 on human keratinocytes in vitro. Mass spectrometric analysis of lesional stratum corneum from $A D$ subjects and IL-13 Tg mice revealed an increased proportion of short-chain ( $N-14: 0$ to $N$-24:0) NS ceramides, sphingomyelins, and 14:0-22:0 lysophosphatidylcholines (14:0-22:0 LPC) with a simultaneous decline in the proportion of corresponding long-chain species ( $N-26: 0$ to $N$-32:0 sphingolipids and 24:0-30:0 LPC) when compared with healthy controls. An increase in short-chain LPC species was also observed in nonlesional AD skin. Similar changes were observed in IL-4/IL-13-driven responses in $\mathrm{Ca}^{2+}$-differentiated human keratinocytes in vitro, all being blocked by STAT6 silencing with siRNA. RNA sequencing analysis performed on stratum corneum of AD as compared with healthy subjects identified decreased expression of fatty acid elongases ELOVL3 and ELOVL6 that contributed to observed changes in atopic skin lipids. IL-4/IL-13 also inhibited ELOVL3 and ELOVL6 expression in keratinocyte cultures in a STAT6-dependent manner. Downregulation of ELOVL3/ELOVL6 expression in keratinocytes by siRNA decreased the proportion of long-chain fatty acids globally and in sphingolipids. Thus, our data strongly support the pathogenic role of type 2 immune activation in AD skin lipid metabolism.
\end{abstract}

Authorship note: $\mathrm{EB}$ and $\mathrm{EC}$ contributed equally to this work.

Conflict of interest: DYML has consulted for Sanofi and Regeneron.

Submitted: October 12, 2017 Accepted: January 18, 2018 Published: February 22, 2018

\section{Reference information:} JCI Insight. 2018;3(4):e98006. https://doi.org/10.1172/jici. insight.98006.

\section{Introduction}

Atopic dermatitis $(\mathrm{AD})$ is a serious systemic disease with lesional skin manifestations that severely impact the quality of life of affected patients (1-3). It is currently well accepted that $\mathrm{AD}$ is linked to dysregulated and overactivated type 2 immune response in the skin, with a special role for IL-4/IL-13-driven signaling in $\mathrm{AD}$ pathogenesis $(2,4)$. This type 2 hyperactivation blocks terminal differentiation of skin keratinocytes and formation of a mature stratum corneum. An intact skin barrier is required to protect the human body from water loss and to prevent the penetration of allergens and pathogens into the skin and underlying tissues. Lipids play an important role in the formation of a fully functional stratum corneum. In a healthy human epidermis, lipids are primarily composed of ceramides, sterols, and free fatty acids, with minimal presence of other lipids that usually represent the majority of lipids in any other tissue and in the circulation (5). In comparison with other skin lipids, ceramides receive special attention, as their content and unique composition is thought to largely determine the efficiency of protective barrier properties of the skin. In particular, human skin ceramides contain an unusually high proportion of very long chain fatty acids and have substantial quantities of unique highly hydrophobic $\omega$-esterified (EOS) ceramides. Several reports have already documented the decrease in the chain length of ceramide-associated fatty acids in the lesional and nonlesional skin of AD patients (6-9). However, only minimal information is available about other lipids potentially associated with AD development. Currently, there is no understanding of what leads to lipid changes during $\mathrm{AD}$ pathogenesis and how one could affect $\mathrm{AD}$ severity or even prevent its development by targeting lipid metabolic processes to preserve or restore normal skin lipid composition. 
In the current work, we have begun to answer mechanistic questions that unveil the link between type 2 immune hyperactivation and lipid changes in the human skin. As part of this study, we have used quantitative and semiquantitative approaches in processing mass-spectrometric data with data normalization per protein content in collected skin tape strips that strongly strengthen the validity of the obtained information. Our study has identified a global shift in all studied epidermal lipids toward molecular species with shorter-chain fatty acids that was recapitulated in the model of IL-13-driven AD skin lesion development in Tg mice. Further RNA sequencing (RNA-seq) analyses performed on human skin tape strips have identified a decreased expression of elongases 3 and 6 (ELOVL3 and ELOVL6) in AD subjects that take part in the process of fatty acid elongation and form very long-chain fatty acids; therefore, the decrease in ELOVL3/6 expression can potentially determine the observed global shift toward short-chain fatty acids in all studied lipid classes. Finally, we have demonstrated the ability to model some aspects of lipid modifications observed in human AD using human keratinocyte differentiation and IL-4/IL-13 treatment in vitro. Keratinocyte culture experiments revealed the inhibition of ELOVL3 and ELOVL6 expression by IL-4/ IL-13 in a STAT6-dependent manner. Downregulation of ELOVL3 and ELOVL6 expression by siRNA shifted the global fatty acid profile toward short-chain species and decreased the relative proportion of longchain species in several lipid classes. Thus, here we set the foundation for an understanding of how exaggerated type 2 signaling globally affects lipid composition in the epidermis and further defines the specific link between $\mathrm{AD}$ lesion development and changes in epidermis lipidome.

\section{Results}

Human AD skin has a characteristic decrease in very long-chain fatty acids within several classes of lipids. It was previously demonstrated that ceramides in the stratum corneum of $\mathrm{AD}$ skin undergo reduced chain length elongation of their $N$-linked fatty acid that can contribute to their total diminished hydrophobicity (10). To see if this is a global characteristic of $\mathrm{AD}$ skin lipids rather than a ceramide-specific phenomenon, we analyzed stratum corneum ceramides, as well as sphingomyelin (SM) and lysophosphatidylcholine (LPC) molecular species by high-performance liquid chromatography electrospray ionization tandem mass spectrometry (UHPLC-ESIMS/MS). In the current study, our analysis of skin tape strips from 25 normal controls, $30 \mathrm{AD}$ nonlesional skin samples, and 15 matching $\mathrm{AD}$ lesional skin samples demonstrated that $\mathrm{AD}$ is characterized by a global reduction in lipid molecular species with long-chain (>24 carbons) fatty acids. This phenomenon was visible not only in ceramides (in particular, in ceramides with C18 sphingosine as their sphingoid base and nonhydroxy fatty acids, NS ceramide), but also in SMs and particularly, in LPCs (Figure 1). The decline in long-chain NS ceramide molecular species (C24-C28) was found to be modest (Figure 1A), similar to SMs (C26-C28) (Figure 1B). In contrast, LPCs demonstrated a profound downregulation of molecular species with fatty acids ranging from $\mathrm{C} 22-\mathrm{C} 30$ carbon atoms (Figure 1C) that was never reported before. Simultaneously, all studied lipids demonstrated a notable increase of molecular species with short-chain fatty acids (C16-C20). This increase was visible for NS ceramides $N$-acylated with C16-C22 fatty acids, SM $N$-acylated with palmitic acid, and LPCs with C16-C18 fatty acids (Figure 1). Strikingly, changes in LPC molecular species were markedly visible not only in stratum corneum collected from $\mathrm{AD}$ lesional skin, but also in nonlesional skin of $\mathrm{AD}$ patients. Furthermore, the volcano plot of changes in relative percentage of analyzed lipids within their corresponding subclasses revealed additional groups of ceramides with C20- and C22-sphingosine with $\alpha$-hydroxy fatty acids that demonstrated significant shifts in their proportion of short-chain and long-chain $\alpha$-hydroxy fatty acid-containing species in nonlesional skin from AD patients. This shift towards increase in short-chain and decrease in long-chain $\alpha$-hydroxy fatty acid-containing species of ceramides with C20- and C22-sphingosine was also present in lesional skin (Figure 2 and Supplemental Table 1; supplemental material available online with this article; https://doi.org/10.1172/jci.insight.98006DS1).

Of note, total absolute amounts of SMs (Supplemental Figure 1B) and NS ceramides (Supplemental Figure 1A) were substantially increased in AD lesional skin when normalized per total sample protein content, while total LPC content normalized per total sample protein content did not change due to simultaneous up- and downregulation of individual LPC molecular species (Supplemental Figure 1C).

In view of the fact that stratum corneum lipids in $\mathrm{AD}$ skin have a clear shift in fatty acids associated not only with sphingolipids, but also with glycerolipids toward the prevalence of species with short-chain fatty acids, we analyzed skin tape strips collected from these patients by RNA-seq for the expression of enzymes that are involved in fatty acid elongation, such as the elongation of long-chain fatty acid family members 1-7 (ELOVL1-7). Figure 3 demonstrates that lesional AD skin has dimin- 
A

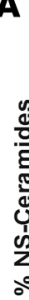

NS-Ceramides

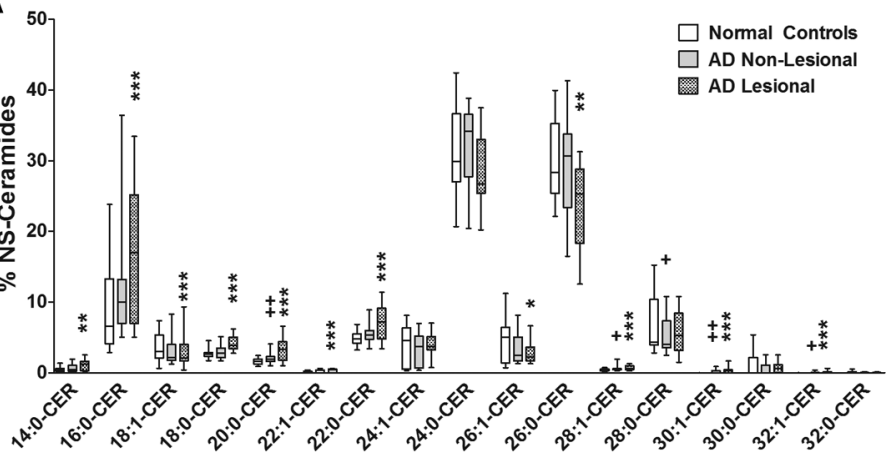

Sphingomyelins

\section{B}

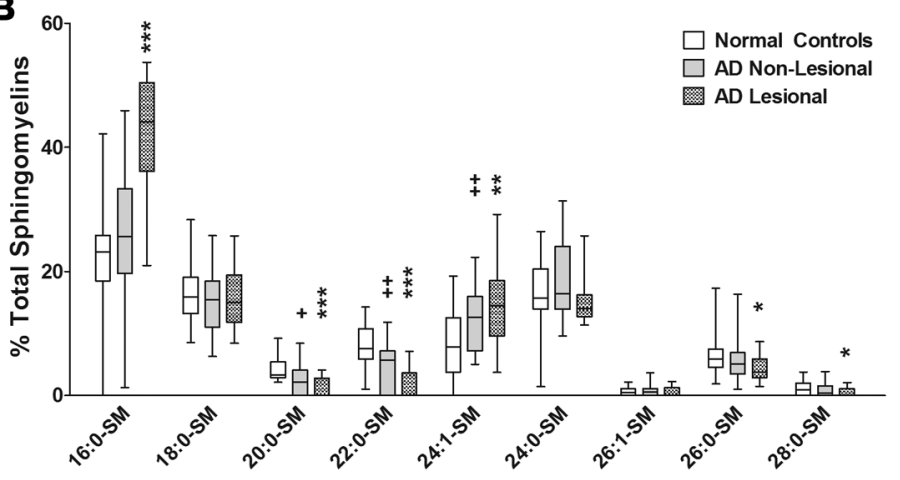

C

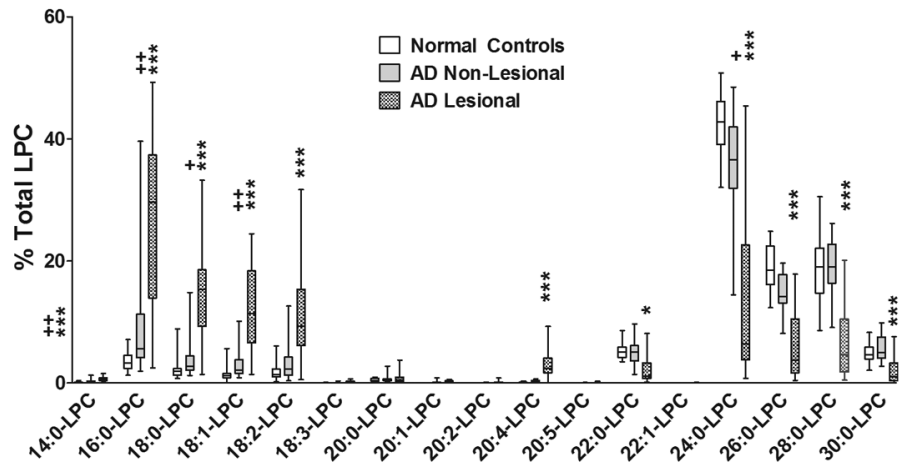

Figure 1. The effect of skin atopy on stratum corneum lipids. Changes in relative level of short- and long-chain molecular species in ceramides (A), sphingomyelins (B) and lysophosphatidylcholines (C) in stratum corneum of atopic dermatitis (AD) patients as compared with skin of normal control subjects. Each lipid molecular species was quantified by targeted liquid chromatography electrospray ionization tandem mass spectrometry (LC-ESI-MS/MS) and normalized by sample total protein content, and data were expressed as relative percentage within each lipid subclass. Data are presented as box-and-whisker plot, with whiskers showing minimum and maximum values. ${ }^{*}+P<0.05,{ }^{* *++} P<$ $0.01,{ }^{* *},+++P<0.001$ as compared with normal control subjects using 2-tailed Student's $t$-test. AD lesional skin $(n=15), A D$ nonlesional skin $(n=30)$, normal control skin $(n=25)$.

ished expression of ELOVL3 and ELOVL6. ELOVL3 is responsible for the formation of long-chain (C18-C24) fatty acids from their short-chain precursors, while ELOVL6 forms short-chain fatty acids (C12-C18). At the same time, the expression of ELOVL1 and ELOVL4 that form predominantly very long-chain fatty acids was increased. Thus, RNA-seq analysis of human tape strips revealed a shift in the expression pattern of enzymes that elongate short- and long-chain fatty acids in lesional AD skin. At the same time, RNA-seq analysis of skin tape strip samples demonstrated increased expression of IL-13 in nonlesional AD skin (Supplemental Figure 2A) and more so in lesional AD skin, which was also accompanied by the upregulation of IL-4R expression in these samples (Supplemental Figure 2B).

Lipid changes in skin tape strips from $I L-13 \mathrm{Tg}$ mice recapitulate lipid phenotype observed in human AD lesional skin. Skin specific inducible K5-tTA-IL-13 Tg (IL-13 Tg) mice have been developed by Tao Zheng's (Yale University) laboratory $(11,12)$. In these mice, IL-13 expression is driven by a keratinocyte-specific promoter upon removal of doxycycline (DOX). As a result, these mice spontaneously develop severe atopic skin lesions 2-4 months after the induction of IL-13 expression in the skin and therefore provide a good model for $\mathrm{AD}$ development in humans. We were able to analyze skin tape strip samples from 6 animals that developed AD lesions, 8 nonlesional areas from animals that either developed skin lesions (6 animals) or did not develop lesions within 3 months after the induction of IL-13 expression ( 2 animals), and 10 WT (noninduced) controls. The analysis of lipids in tape strips collected from lesional areas of induced IL-13 Tg animals recapitulated changes in lipid molecular species that we observed in human lesional AD stratum corneum samples (Figure 4). Notably, both SMs and NS ceramides demonstrated a substantial increase in relative content of short-chain species (in particular, 16:0 species) with a decline in the relative content of long-chain species (especially in $N$-26:0-NS ceramide and 26:0 SM) in the lesional skin tape strip samples of IL-13 Tg mice, similar to what we observed in human AD lesional stratum corneum. Furthermore, lesional stratum corneum from IL-13 Tg mice demonstrated changes in LPC molecular species involving an increase in shortchain species (18:0 LPC) and a decline in relative percentage of the long-chain LPC species (24:0 LPC and 26:0 LPC). Thus, the mouse model of IL-13-driven AD development demonstrated changes almost identical to human $\mathrm{AD}$ in the profile of molecular species in several lipid classes.

To confirm that the formation of skin lesions in IL-13 Tg mice is linked to IL-13 production, IL-13 was quantified by reverse transcription PCR (RT-PCR) in skin biopsies obtained from lesional as well as nonlesional areas of the skin in the induced animals and from biopsies obtained from noninduced control animals. Figure 5A demonstrates a significant induction of IL-13 level in lesional areas of mouse 
Human AD Non-Lesional vs Healthy CTR Non-Lesional Skin

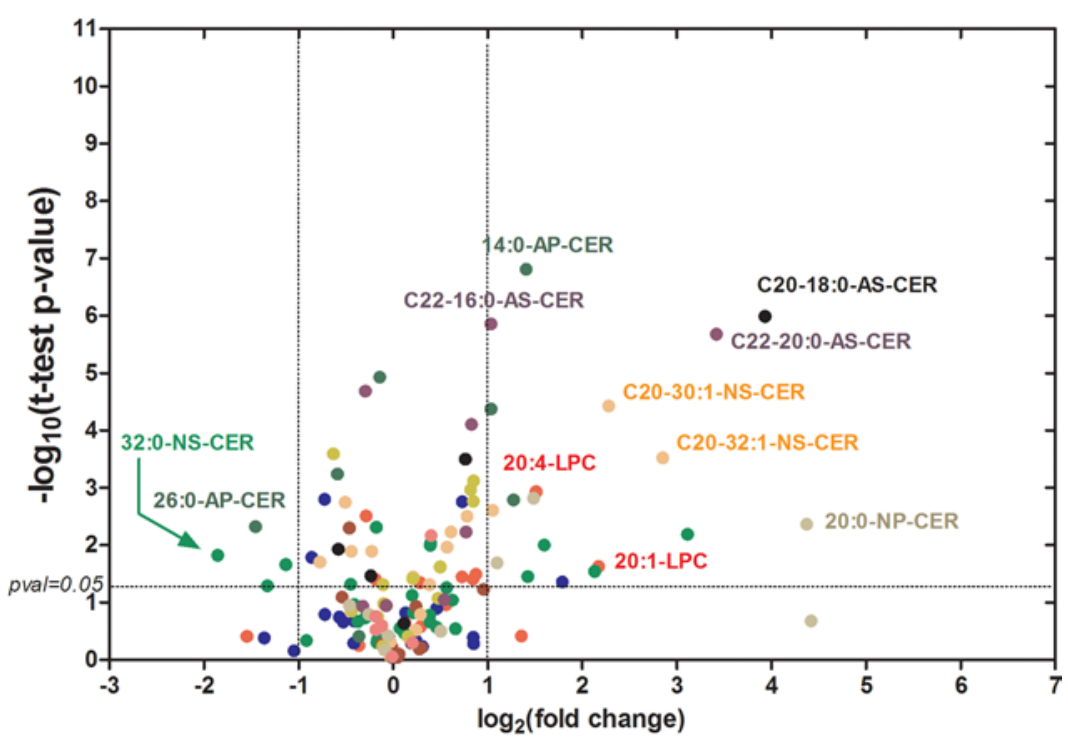

Human AD Lesional vs Healthy CTR Non-Lesional Skin
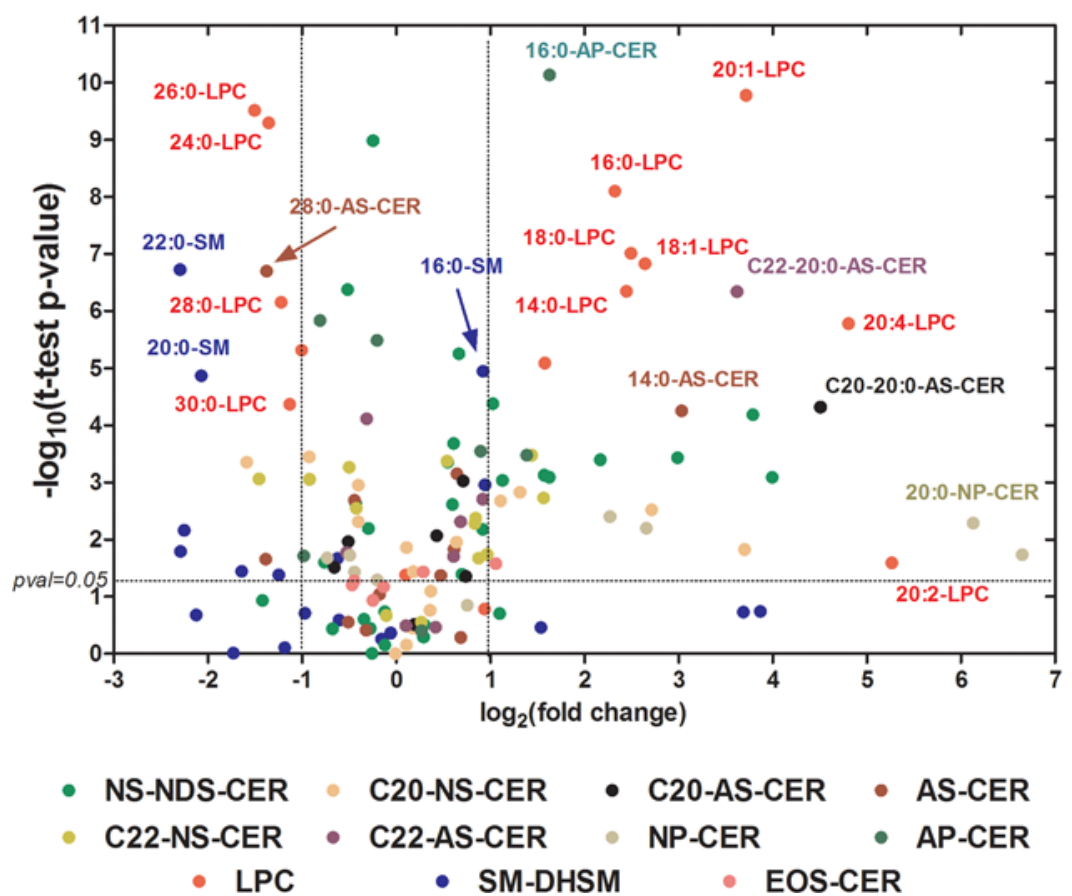

Figure 2. Volcano plot representation of lipid changes associated with atopic dermatitis in stratum corneum. Volcano plots of changes in relative percentage in molecular species of lipids from selected individual lipid subclasses in nonlesional (A) and lesional (B) stratum corneum of atopic dermatitis (AD) patients versus normal control (CTR) subjects. Each lipid molecular species was quantified by targeted or semitargeted liquid chromatography electrospray ionization tandem mass spectrometry (LC-ESI-MS/MS), normalized by sample total protein content, and data were expressed as relative percentage within each lipid subclass. Note that lysophosphatidylcholine (LPC) molecular species demonstrate the most striking reciprocal shift in the long-chain and short-chain molecules in lesional $A D$ skin. AD lesional skin $(n=15), A D$ nonlesional skin $(n=30)$, normal control skin $(n=25)$.

skin and its minimal level in nonlesional skin from induced animals and in the skin of control noninduced animals. RT-PCR analysis of mouse skin biopsies also revealed significant downregulation of ELOVL3 and ELOVL6 mRNA in the lesional skin of IL-13 Tg mice (Figure 5, B and C).

The effects of type 2 cytokines on sphingolipid metabolism in cultured primary human keratinocytes are STAT6 dependent. Keratinocytes are the major cells in the skin that form the stratum corneum and its lipids. To model the effects of type 2 cytokines on keratinocyte lipid metabolism, we used primary human keratinocytes grown in a submerged culture, differentiated in the presence of $1.3 \mathrm{mM} \mathrm{CaCl}_{2}$, and treated in the presence or absence of IL- 4 and IL-13 during the entire differentiation period.

Upon differentiation, keratinocyte sphingolipids undergo substantial changes. Notably, the relative proportion of long-chain ( $N-24: 0$ to $N$ 28:0) molecular species of ceramides preferentially increases over short-chain $(N-14: 0$ to $N-22: 0)$ species (Figure 6A) that corresponds to prevailing presence of long-chain ceramides in mature stratum corneum. When keratinocytes were treated with type 2 cytokines during differentiation, cytokine presence had complex effect on ceramides, as well as other sphingolipids. In particular, IL-4/IL-13 treatment led to a substantial increase in ceramide and SM content, with some preferential increase in short-chain species (sphingoid bases $N$-acylated with C16-C20 fatty acids) (data not shown). However, LPC levels and their relative composition in differentiated keratinocytes did not change significantly upon type 2 cytokine treatment (data not shown).

STAT6 is a master transcriptional regulator of IL-4/IL-13-induced signaling in cells. To test if STAT6 controls IL-4/IL-13-induced changes in sphingolipids, we transfected human keratinocytes with STAT6 siRNA or control nontargeting siRNA and differentiated keratinocyte cultures for 5 days in the presence of $1.3 \mathrm{mM} \mathrm{CaCl}_{2}$. Some cells were also treated with IL-4/IL-13 during the entire differentiation period. As shown in Figure 6, B and C, STAT6 silencing completely blocked the IL-4/IL-13-induced upregulation in ceramide and SM levels. Of note, neither IL-4/IL-13 treatment nor STAT6 silencing had affected the LPC content in cells (Figure 6D). Significant inhibition of STAT6 mRNA expression by STAT6 siRNA in keratinocyte cultures was confirmed by RT-PCR (Figure 7, A and D). 

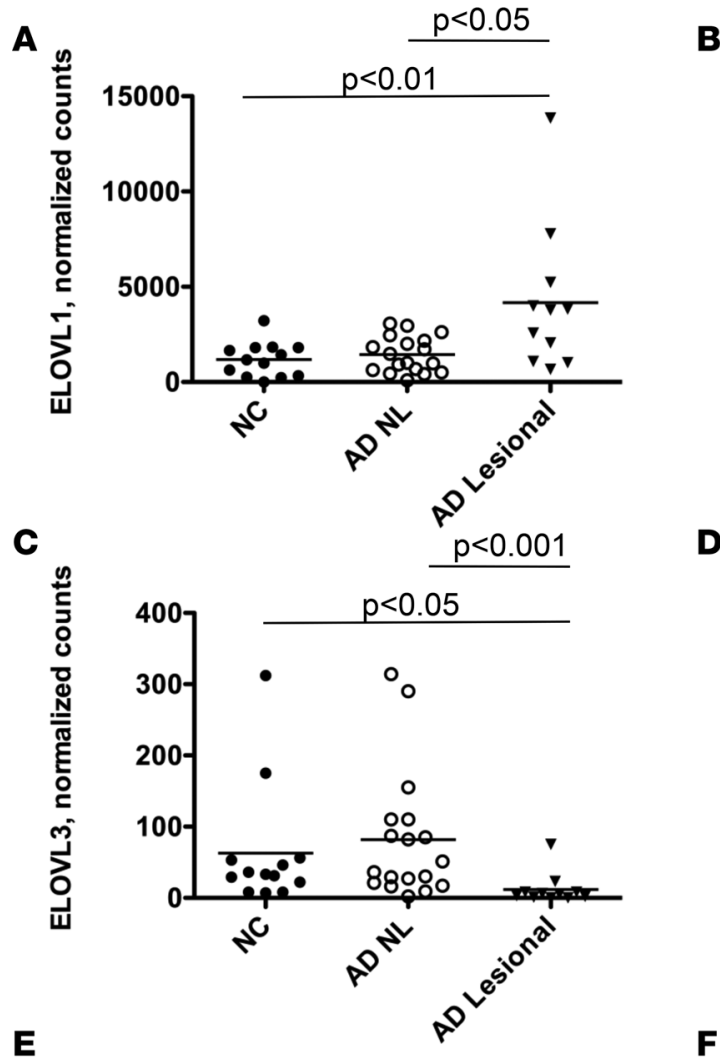

E

G
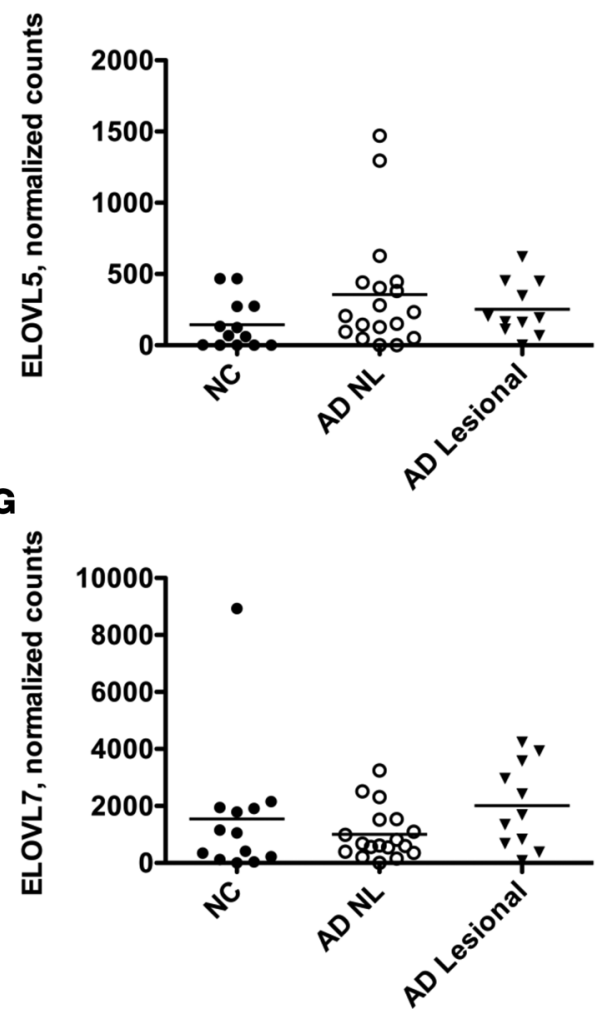

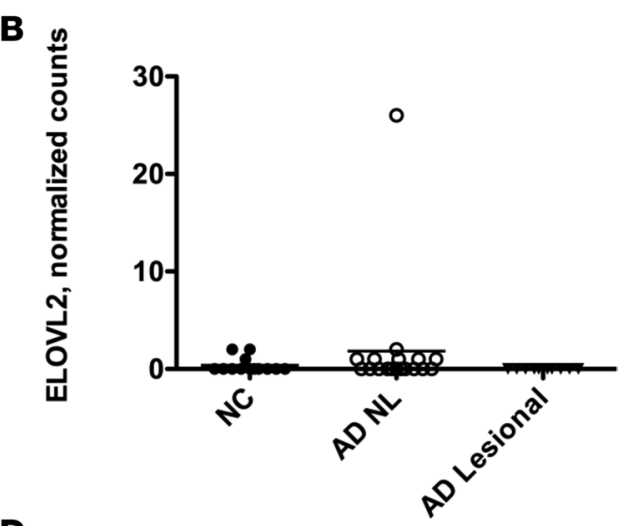

Figure 3. Expression of elongation of long-chain fatty acids family member 3 and 6 (ELOVL3 and ELOVL6) enzymes is decreased in atopic skin. Expression pattern for elongation of long-chain fatty acids family member 1-7 (ELOVL1, ELOVL2, ELOVL3, ELOVL4, ELOVL5, ELOVL6, ELOVL7) (A-G) in human stratum corneum as detected by RNA-seq analysis of skin tape strip RNA samples collected from atopic dermatitis (AD) patients and normal controls (NC). Note that the expression of ELOVL3 and ELOVL6 is decreased in lesional AD skin (both elongases take part in the formation of long-chain fatty acids). AD lesional skin $(n=11), A D$ nonlesional skin $(n=18)$, NC skin ( $n$ $=13$ ). One-way ANOVA was used for statistical analysis.
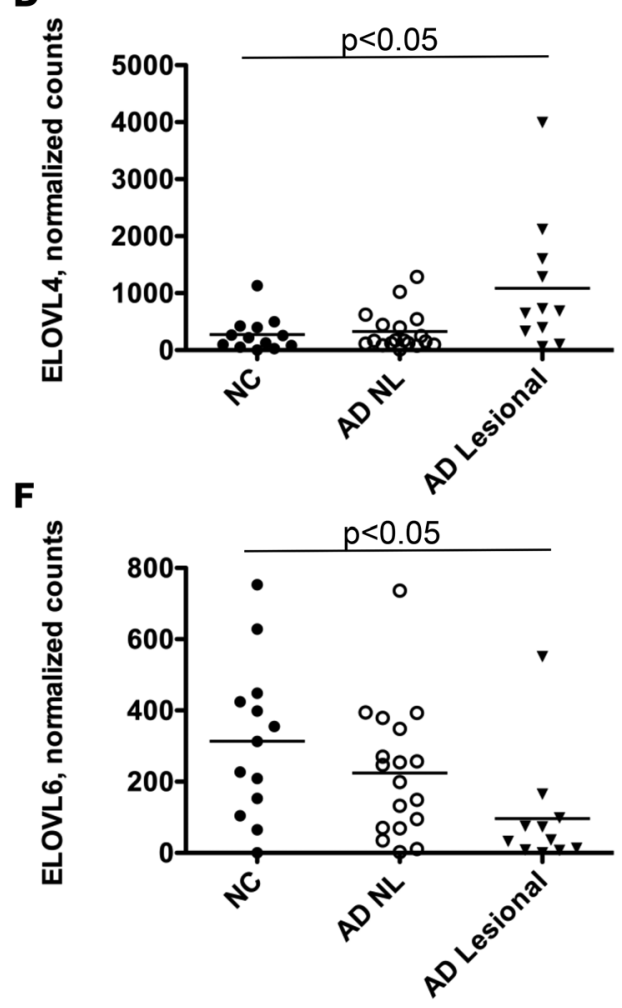

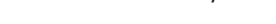


A

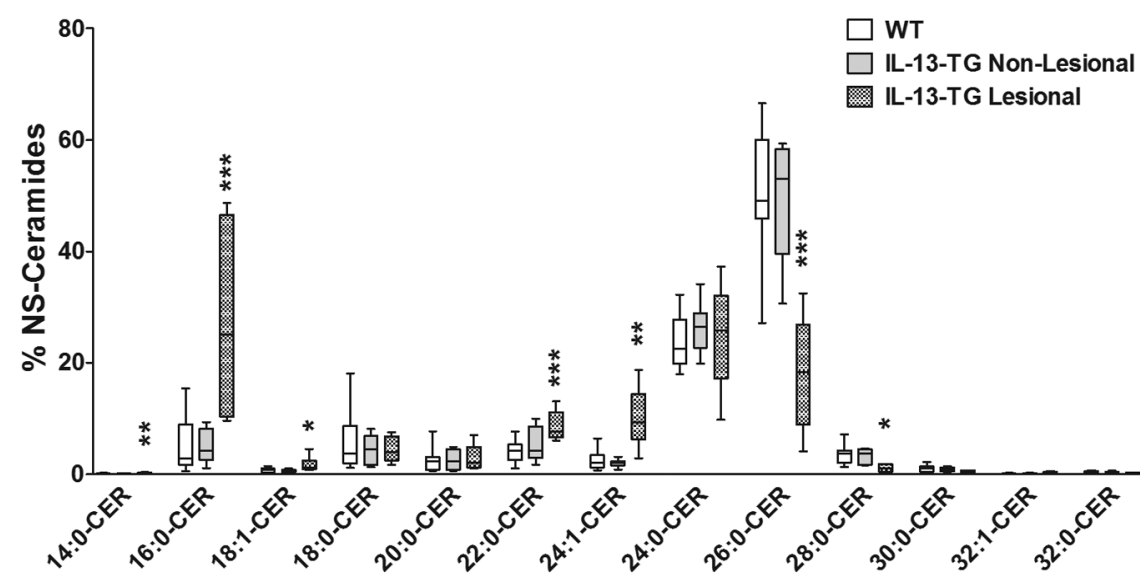

B

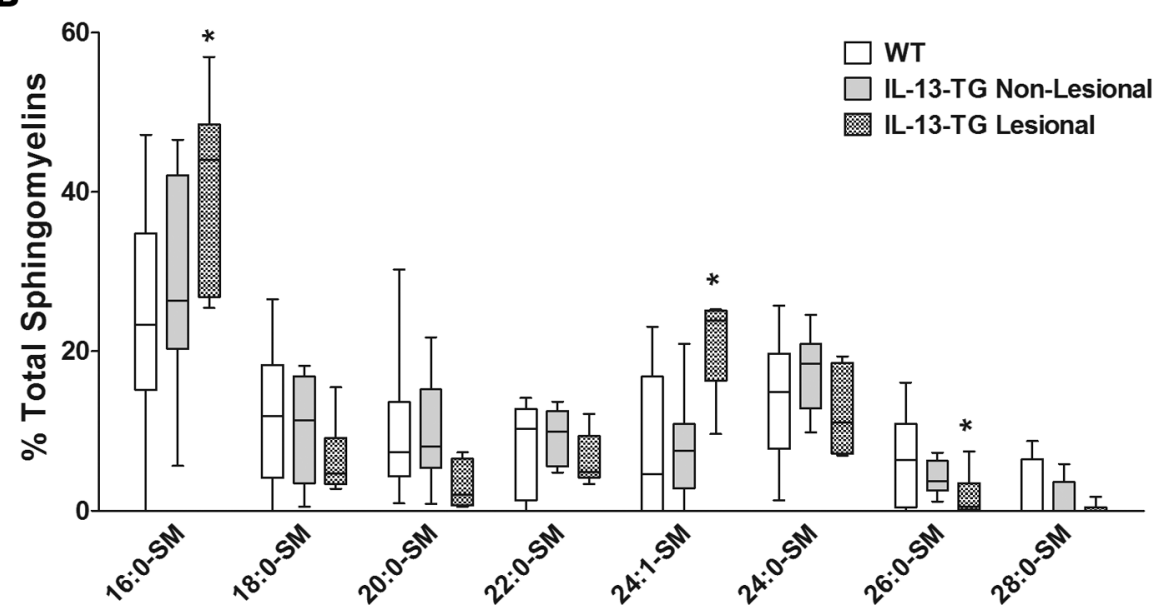

C

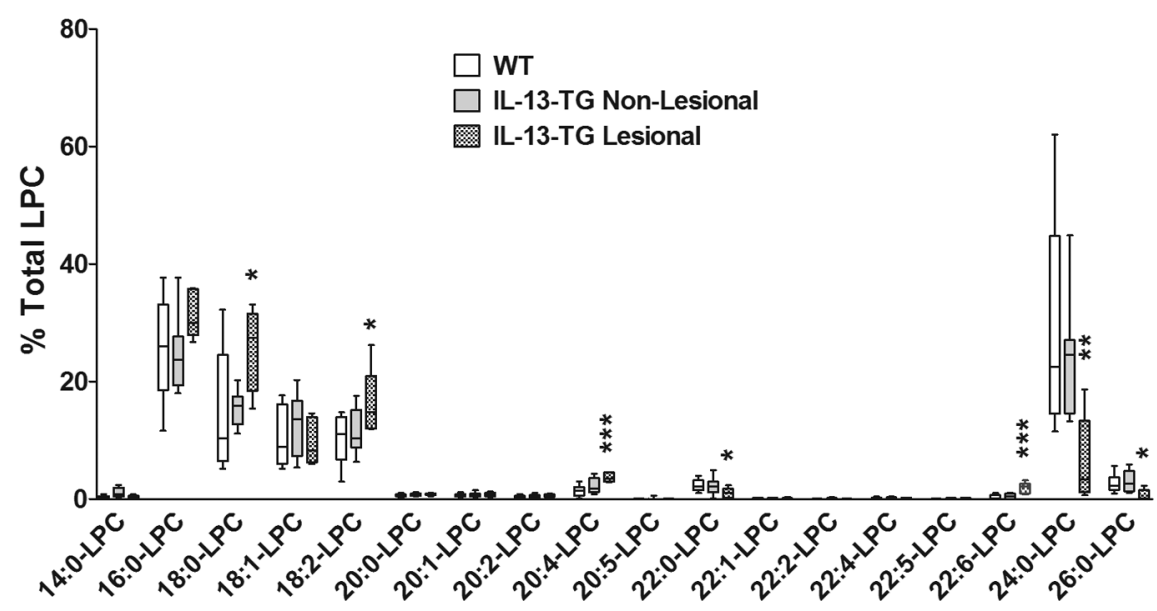

Figure 4. IL-13 driven lesion development in mouse skin profoundly affects stratum corneum lipids. Changes in relative level of short- and long-chain molecular species in NS ceramides with C18 sphingosine (A), sphingomyelins (B), and lysophosphatidylcholines (C) in stratum corneum from nonlesional and lesional skin of $\mathrm{IL}-13 \mathrm{Tg}$ mice and their littermate controls. IL-13 Tg mice spontaneously develop skin lesions. Tape strips were collected from both lesional and nonlesional areas of mouse skin. Each lipid molecular species was quantified by targeted liquid chromatography electrospray ionization tandem mass spectrometry (LC-ESI-MS/MS) and normalized by sample total protein content, and data were expressed as relative percentage within each lipid subclass. Data are presented as box-and-whisker plot, with whiskers showing minimum and maximum values. ${ }^{*} P<0.05,{ }^{* *} P<0.01,{ }^{* *} P<0.001$ versus WT littermate control as determined using 2-tailed Student's $t$ test. IL-13 Tg lesional skin samples $n$ $=6$, IL-13 Tg nonlesional skin samples $n=8, \mathrm{WT}$ control mice $n=10$.

of STAT6 expression restored mRNA levels of both ELOVL3 and ELOVL6 (Figure 7, B, C, and E). However, ELOVL6 mRNA expression was suppressed by IL-4/IL-13 in the presence of STAT6 siRNA at the later time point (Figure 7F). Regulation of ELOVL3 expression by IL-4/IL-13 was confirmed by immunostaining (Figure 7G), as decreased levels of ELOVL3 were observed in differentiated keratinocytes treated with IL-4/IL-13, while STAT6 inhibition restored ELOVL3 expression in IL-4/IL-13treated keratinocytes. Very low ELOVL3 expression was observed in ELOVL3 siRNA transfected 


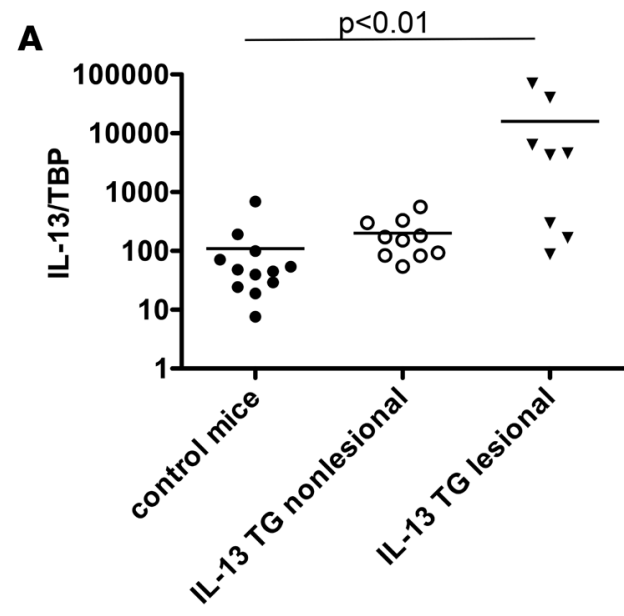

B $\quad p<0.05$

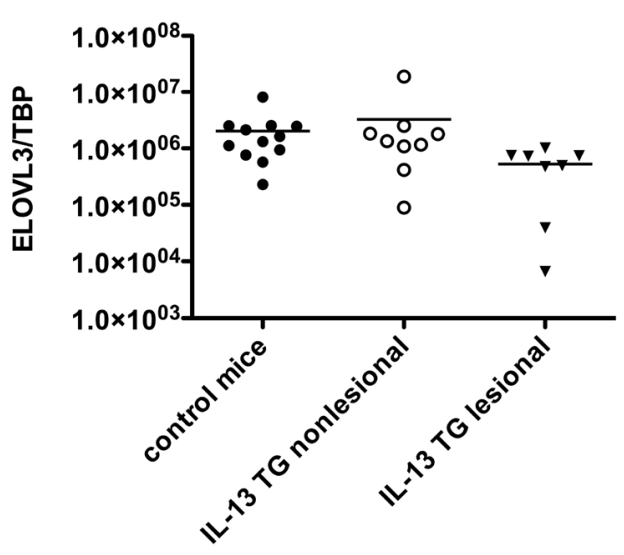

C $\quad p<0.05$

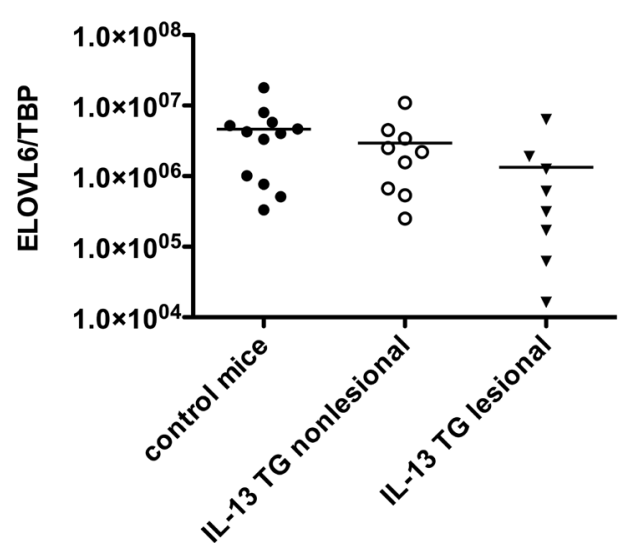

Figure 5. IL-13 downregulates skin expression of elongation of long-chain fatty acids family member 3 and $\mathbf{6}$ (ELOVL3 and ELOVL6) enzymes in mice. Expression of IL-13 (A), elongation of long-chain fatty acids family member 3 (ELOVL3) (B), and elongation of long-chain fatty acids family member 6 (ELOVL6) (C) in skin of IL-13 Tg mice and control animals as detected by realtime PCR. An increased expression of IL-13 mRNA and significant downregulation of ELOVL3 and ELOVL6 mRNA expression was observed in lesional skin of IL-13 Tg mice. IL-13 Tg lesional skin samples $(n=8)$, IL-13 Tg nonlesional skin samples $(n=10)$, WT control mice $(n=12)$.

keratinocytes. No effects of ELOVL6 siRNA on the expression of ELOVL3 in keratinocytes was found (Figure 7G). Thus, these data demonstrate the interrelationship between the expression of STAT6 and elongases ELOVL3 and ELOVL6.

Simultaneously, downregulation of ELOVL3 and ELOVL6 expression with siRNA upregulated short-chain fatty acids globally, as shown by the analysis of total fatty acids in all glycerolipids, increased relative proportion of LPC with palmitic acid, and decreased relative levels of long-chain species in NS ceramides and SMs (Supplemental Figure 4).

\section{Discussion}

$\mathrm{AD}$ is an immune system-driven disease associated with skin barrier defects (2). The critical importance of type 2 immune pathway activation in the pathogenesis of $\mathrm{AD}$ has recently been demonstrated by the significant clinical improvement of moderate to severe $\mathrm{AD}$ following treatment with biologics that interfere with IL-4/IL-13 action, such as antibodies directed to the $\alpha$ subunit of the IL-4 receptor (Dupilumab) (13). The loss of skin barrier function is the hallmark of $\mathrm{AD}$, and lipids play a fundamental role in forming the proper hydrophobic barrier in stratum corneum that reduces transepidermal water loss. In $\mathrm{AD}$ skin, however, this lipid-derived barrier is not properly formed, and very little is known about what drives the lipid changes in $\mathrm{AD}$ skin (14).

Skin lipids are unique in many aspects and provide a pivotal role to form a proper skin barrier. Healthy epidermis has lipids that are mostly composed of ceramides, free fatty acids, and cholesterol $(10,15)$, with very little presence of other lipids. Ceramides in the skin are particularly complex and are represented by at least 12 groups based on sphingoid base and type of fatty acid attached to it (Supplemental Figure 3). A very specific, highly hydrophobic group of ceramides, called EOS ceramides, is present only in the skin. Fatty acids are also unique in skin ceramides, as they are unusually very long (up to C38) and hydrophobic; this also contributes to the overall requirement for a highly rigid and hydrophobic structure to provide an efficient barrier. Several groups of investigators have already reported that lesional and, in some cases, nonlesional skin of $\mathrm{AD}$ patients has decreased proportion of EOS ceramides and other ceramides with very long-chain fatty acids (6-9). Such changes in skin lipid composition result in aberrant lipid organization in the lipid layers and positively correlate with the degree of transepidermal water loss in $\mathrm{AD}$ skin $(16,17)$.

It has previously been demonstrated that genetic abnormalities in the expression of filaggrin (FLG) protein result in the aberrant lipid delivery into the lamellar bodies $(16,18)$. It is not clear which exact mechanism would define such an effect the abnormal transport of complex lipids into lamellar bodies or abnormal activity of enzymes responsible for their degradation into ceramides. However, FLG mutations can not account for lipid abnormalities in all $\mathrm{AD}$ patients, as only a minority of AD patients of European descent have FLG mutations (19). At the same time, our research group has demonstrated that type 2 cytokines can also significantly inhibit FLG expression in keratinocytes (20), and such reduced levels of FLG can also affect lipid assembly in lamellar bodies. Importantly, another research group has demonstrated that changes in stratum corneum lipid properties correlate with disease severity but are independent of FLG mutations (6). Cole and coworkers (21) performed RNA-seq analysis of nonlesional skin samples from AD patients and healthy controls; they then stratified the analysis of changes in $\mathrm{AD}$ transcriptome based on FLG gene mutations. They found that patients with no FLG mutations had significant changes in the expression of enzymes involved in the metabolism and synthesis of lipids. However, no information is currently available on signaling mechanisms and metabolic pathways that promote these changes in skin ceramides. 
A

\section{Selected Ceramides}

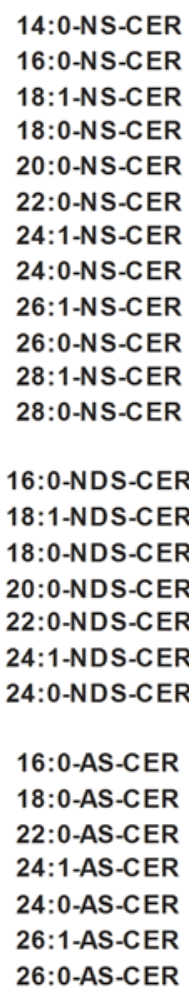

14:0-NS-CER

16:0-NS-CER

S-CER

20:0 NS-C

22:0-NS-CER

24:1-NS-CER

24:0-NS-CER

26:1-NS-CER

26:0-NS-CER

28:1-NS-CER

6:0-NDS-CER

20:0-NDS-CER

22:0-NDS-CER

24:1-NDS-CER

24:0-NDS-CER

16:0-AS-CER

18:0-AS-CER

22:0-AS-CER

24:1-AS-CER

26:1-AS-CER

26:0-AS-CER
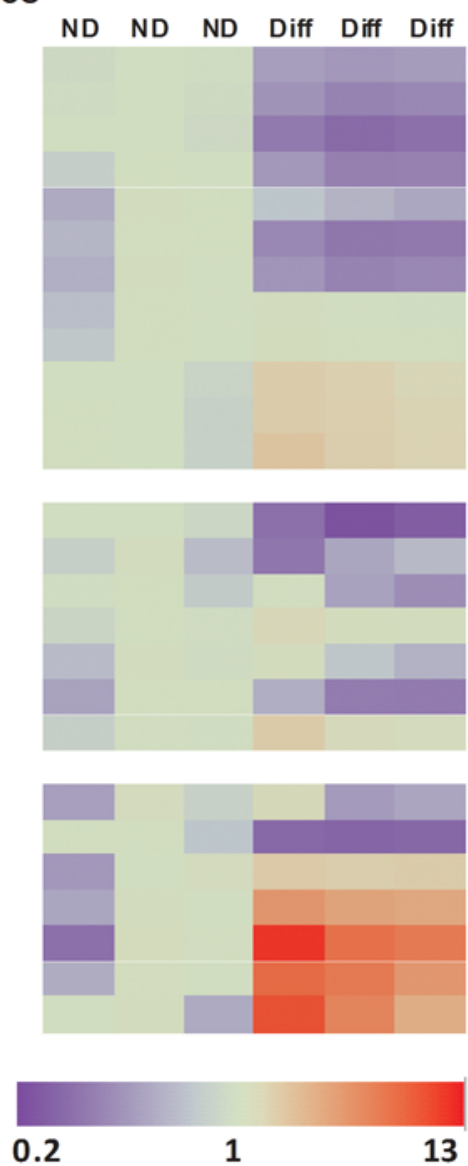

Fold Change
B

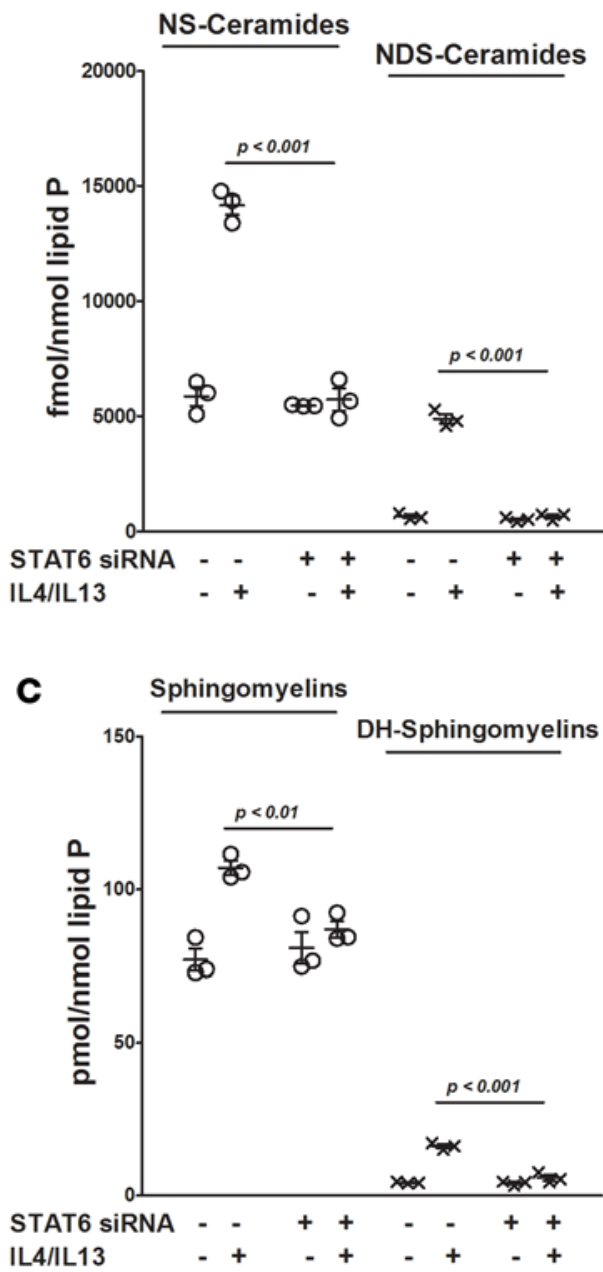

D

Lysophosphatidylcholines

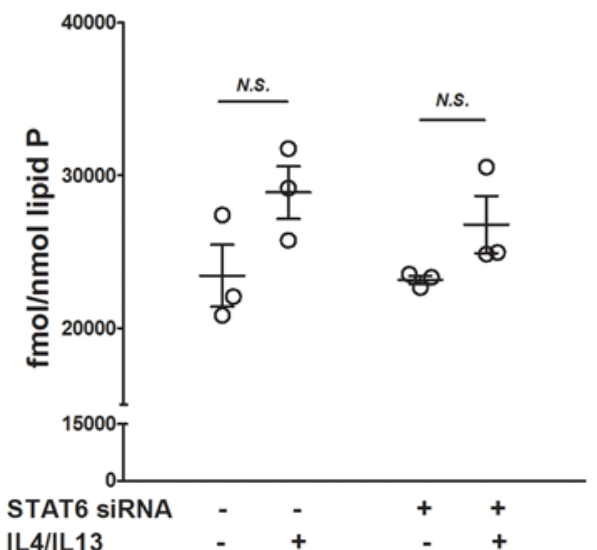

Figure 6. The effect of differentiation and type $\mathbf{2}$ cytokines on keratinocyte lipids. (A) The effect of $\mathrm{Ca}^{2+}$-induced differentiation in vitro on relative proportion of selected ceramides in keratinocytes. Each ceramide molecular species was quantified by targeted liquid chromatography electrospray ionization tandem mass spectrometry (LC-ESI-MS/MS) and normalized by sample total lipid phosphorus content, and data were expressed as relative percentage within each lipid subclass. Each individual data point was expressed relative to an average of nondifferentiated control. Note the decrease in relative content of short-chain ceramides and the increase in long-chain ceramides. STAT6 controls the effect of IL-4/IL-13 on NS ceramides with C18 sphingosine (B) and sphingomyelins (C) in differentiated keratinocytes. No differences in lysophosphatidylcholines (LPC) are observed in the keratinocyte model (D). Keratinocytes were differentiated in the absence or presence of IL-4/IL-13. Keratinocytes were treated with STAT6 siRNA during a 5-day differentiation period. Lipids were quantified by LC-ESI-MS/MS and normalized by total lipid phosphorus content $(n=3)$. One from 2 typical experiments is shown; each treatment condition done in triplicates. Two-tailed Student's $t$ test was used for statistical analysis. 
3 days of $\mathrm{Ca}^{2+}$ differentiation
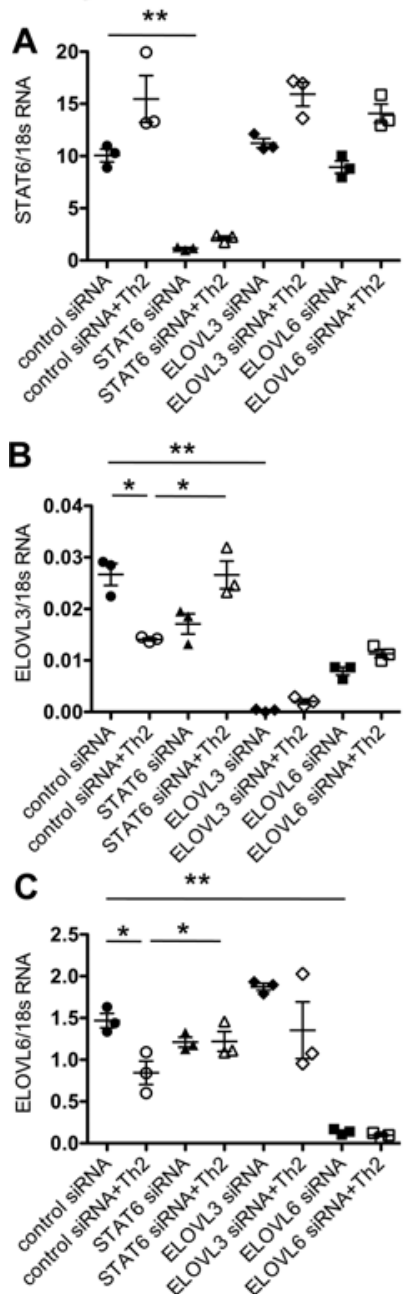

5 days of $\mathrm{Ca}^{2+}$ differentiation
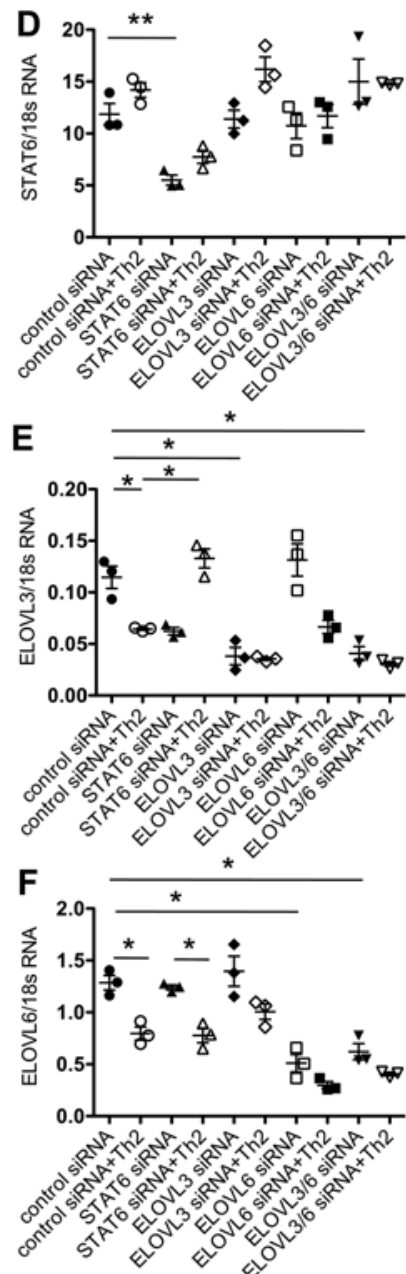

G
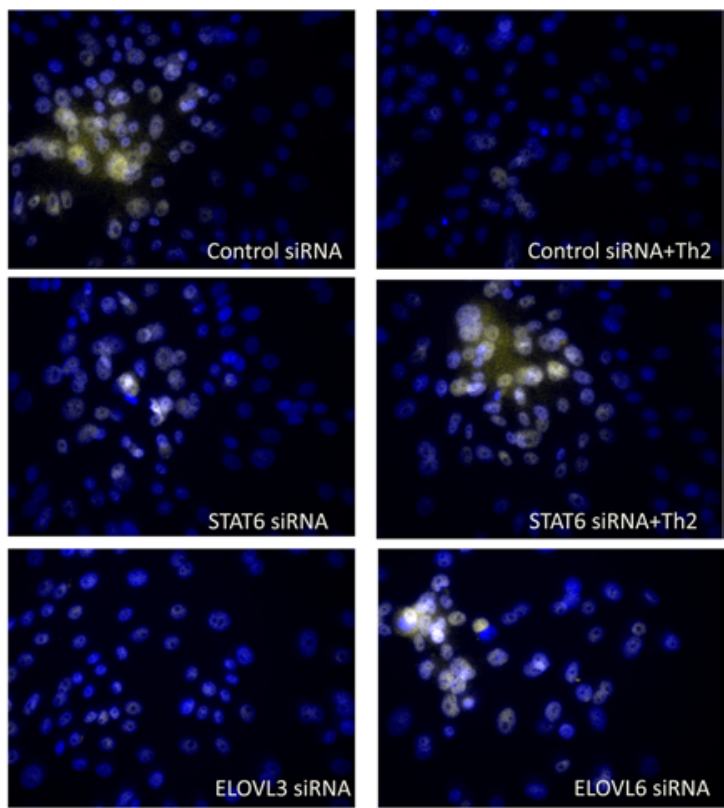

Figure 7. Type $\mathbf{2}$ cytokine regulation of elongation of long-chain fatty acids family member $\mathbf{3}$ and $\mathbf{6}$ (ELOVL3 and ELOVL6) expression in primary human keratinocytes. Keratinocytes were differentiated in the absence or presence of IL-4/IL-13. Keratinocytes were transfected with control siRNA, STAT6 siRNA, ELOVL3 siRNA, ELOVL6 siRNA, or ELOVL3/ELOVL6 siRNA and underwent $\mathrm{Ca}^{2+}$ differentiation for the period of time indicated. The expressions of STAT6 mRNA (A and D), ELOVL3 mRNA (B and E), and ELOVL6 (C and F) were analyzed. (G) ELOVL3 immunostaining in keratinocytes. ELOVL3 staining, yellow; DAPI, cell nuclei staining, blue. Magnification, 250x. ${ }^{*} P<0.05$, ${ }^{* *} P<0.01$, 2-tailed Student's $t$ test $(n=3)$.

Our finding that the shift in molecular species toward shorter-chain molecules in $\mathrm{AD}$ stratum corneum happens not only in ceramides, but also in SMs and especially in LPCs clearly points to a global change in fatty acid elongation. In contrast to all previous known publications focused on ceramides, we have included the analysis of LPC molecular species in our investigation. Our decision to look at LPC was determined by 2 factors. First, most LPCs are formed from its major metabolic precursor phosphatidylcholine (PC), which is the major gycerophospholipid in cells. Second, and most important, the majority of LPC molecules formed from $\mathrm{PC}$ retain its $s n-1$ fatty acids that are incorporated into glycerol-3-phosphate and then into PC during de novo biosynthesis and, therefore, reflect the global state of fatty acid biosynthesis and elongation rather than remodeling through Lands' cycle (22). Our subsequent RNA-seq analysis performed on tape strips from $\mathrm{AD}$ and healthy subjects provided mechanistic background for the observed lipid changes by identifying decreased expression of ELOVL3 and ELOVL6 in AD lesional skin (Figure 3). We believe that a concomitant increase in the expression of ELOVL1 and ELOVL4 (Figure 3) represents a compensatory response to decreased expression of ELOVL3 and ELOVL6 that only partially corrects for the insufficiency of short-chain substrates provided by ELOVL6 and long-chain fatty acids formed by ELOVL3. Previous meta-analysis combining 4 published $\mathrm{AD}$ transcriptome datasets identified wide lipid abnormalities in $\mathrm{AD}$ transcriptome and correlated type 2 immune activation with downregulation of ELOVL3 and several other enzymes (23). ELOVL3 has 
been previously shown to be important for proper skin formation and functioning. ELOVL3-KO mice exhibit a severe defect in water repulsion and increased transepidermal water loss (24). These mice display a sparse hair coat with a hyperplastic pilosebaceous system and disturbed hair lipid content with exceptionally high levels of eicosenoic acid (20:1). In the skin of these mice, the triglyceride fraction with fatty acids longer than 20 carbon atoms was almost undetectable (24). In addition, it has been shown that ELOVL3-synthesized C18:1 and C20:1 fatty acids act as agonists of $\operatorname{PPAR} \gamma$, the master transcriptional factor regulator involved in lipid metabolism (25). Therefore, it is logical to expect that any change in fatty acid homeostasis due to disregulated ELOVL expression will globally affect lipid metabolism.

Striking similarity of lipid changes in stratum corneum between human AD subjects and induced IL-13 Tg mice strongly supports the fundamental role of type 2 cytokine signaling in modifying lipid metabolism in the skin in a way that decreases the overall hydrophobicity and barrier properties of stratum corneum. It should be pointed out that we have also found a substantial increase in total content of sphigomyelins in stratum corneum (Supplemental Figure 1). The work by our research group has previously demonstrated that IL-4/IL-13-mediated elevation of SM levels in keratinocyte membranes made keratinocytes more susceptible to binding of staphylococcal $\alpha$ toxin, thus enhancing cytolytic effects of $\alpha$ toxin in these cells (26). Therefore, in a clinical setting, the increase in SM content in keratinocytes in skin will favor $S$. aureus virulence, thus contributing to $S$. aureus-induced complications of $\mathrm{AD}$.

Decreased acid sphingomyelinase (aSMase) activity was previously reported in AD skin (27). Our research group has shown that aSMase localizes in punctate granules in differentiated keratinocytes, consistent with its association with lamellar bodies (26). We have observed a reduction in lamellar body formation and diffused aSMase staining in IL-4/IL-13-treated keratinocytes (26). Given an increase in SM levels in $\mathrm{AD}$ skin and in the skin of IL-13 Tg mice and IL-4/IL-13-treated differentiated keratinocytes that we observed in vitro in our current study, further investigations on assembly of aSMase in keratinocyte lamellar bodies and the role of IL-4/IL-13 in this process are warranted.

Of interest, Niemann-Pick patients with severe aSMase deficiency $(<2 \%$ residual activity) demonstrate abnormal permeability barrier homeostasis (i.e., delayed recovery kinetics following acute barrier disruption by tape stripping; ref. 28). Delays in barrier recovery can be overridden by coapplications of topical ceramide, demonstrating that an alteration of the ceramide/SM ratio, rather than just SM accumulation, is likely responsible for the barrier abnormalities that occur with aSMase deficiency. These studies emphasize the importance for enzymatic processing of SM to ceramide by aSMase as a mechanism for generating a portion of the stratum corneum ceramides for permeability barrier homeostasis in the skin (28).

Studies on permeability and biophysics of artificial lipid membranes have shown that short-chain ceramides increase the permeability of the lipid membranes compared with long-chain ceramides (29). Membranes generated from ceramides with long acyl chains formed tightly packed impermeable lipid lamellae (29). Membranes containing $N$-16:0-NS ceramide were significantly more permeable to water (by $38 \%-53 \%$ ), theophylline (by 50\%-55\%), and indomethacin (by $83 \%-120 \%$ ) than those containing the very long $N$-24:0-NS ceramide. $N$-24:0-NS ceramide membranes were more condensed than membranes enriched for $N$-16:0-NS ceramide (30). In the membranes composed of ceramide/free fatty acids/cholesterol sulfate, partial or full replacement of ceramide by SM increased water loss, while the permeability barrier to exogenous compounds was less sensitive to the presence of SM (31). Therefore, a reduction of ceramide chain length and concomitant increase in SM content in AD skin and skin of IL-13 Tg mice may result in enhanced transepidermal water loss and penetration of allergens into the skin.

The submerged culture of keratinocytes provides a very remote view on events happening in the skin. Regardless of all known limitations of such a model, we were able to recapitulate main observations from our in vivo studies using $\mathrm{Ca}^{2+}$-differentiated human keratinocytes in vitro. Most importantly, we have demonstrated the critical importance of STAT6-mediated signaling in conveying the effect of IL-4/IL-13 on keratinocyte sphingolipids (Figure 6). It should be noted that, while promoting changes in ceramides and SM, IL-4/IL-13 did not affect LPC molecular species composition in differentiated keratinocytes. We have also demonstrated inhibition of ELOVL3 and ELOVL6 by IL-4/IL-13 in keratinocyte cultures (Figure 7). Further studies are needed to define a protocol with better balance between differentiation and type 2 immune response signaling. Regardless of current limitations, the in vitro keratinocyte model allows the study of major signaling pathways involved in regulation of sphingolipid metabolism by type 2 cytokines.

Studies from our laboratory and others have demonstrated that IL-4 and IL-13 are important for the pathological events that lead to $\mathrm{AD}$, including the inhibition of expression of epidermal barrier proteins, 
Table 1. Study subject characteristics.

\begin{tabular}{|c|c|c|}
\hline & $\begin{array}{c}\text { AD } \\
n=30\end{array}$ & $\begin{array}{l}\text { Healthy controls } \\
\qquad n=25\end{array}$ \\
\hline Age, years (mean \pm SE) & $38.4 \pm 2.4$ & $34.8 \pm 2.1$ \\
\hline Sex (male/female) & $20 / 10$ & 9/16 \\
\hline $\begin{array}{l}\text { Total serum lgE, kU/l } \\
\text { (mean } \pm \mathrm{SE})\end{array}$ & $3,184 \pm 1,806$ & ND \\
\hline EASI (Mean \pm SE) & $22.7 \pm 4.4$ & NA \\
\hline \multicolumn{3}{|l|}{$\begin{array}{l}\text { Investigator's Global } \\
\text { Assessment }\end{array}$} \\
\hline $2^{A}$ & 5 & NA \\
\hline $3^{\mathrm{B}}$ & 19 & \\
\hline $4^{c}$ & 6 & \\
\hline $\begin{array}{l}\text { Body surface area } \\
\text { covered by lesions, \% }\end{array}$ & $37 \pm 4$ & NA \\
\hline $\begin{array}{l}\text { Positive skin culture for } \\
\text { Staphylococcus aureus }\end{array}$ & 12 of 30 & 2 of 25 \\
\hline
\end{tabular}

ND, not done; NA, not applicable; Amild Investigator's Clobal

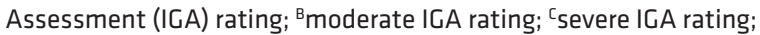
EASI, eczema area and severity index.
FLG, involucrin (IVOL), loricrin (LOR), and others $(20,32)$. The importance of the IL-4/IL-13 pathway in severe $\mathrm{AD}$ was demonstrated by the recent observation that blocking the IL-4R $\alpha$ pathway with Dupilumab leads to significant clinical improvement in severe $\operatorname{AD}(13,33)$.

In summary, our study demonstrates that type 2 cytokines globally change lipid metabolism in the skin and reduce their carbon chain length by altering the expression of a number of enzymes involved in fatty acid elongation and sphingolipid metabolism. These alterations in skin lipidome are detrimental to the skin barrier function and illustrate a potentially novel pathogenic function of IL-4/IL-13 activation in AD.

\section{Methods}

Study subjects. Skin samples were obtained (National Jewish Health) from 30 adult subjects of European descent with active $\mathrm{AD}$ and 25 nonatopic healthy individuals with no personal or family history of atopy and skin diseases. $\mathrm{AD}$ patients were characterized by Investigator's Global Assessment (IGA) score, eczema area and severity index (EASI), and information about the body surface area covered by lesions. Patients had not received topical corticosteroids, topical calcineurin inhibitors, or topical or oral antibiotics for 1 week prior to enrollment. Patients were not treated with systemic immunosuppressive medications for more than 1 month prior to enrollment into this study. The study was approved by the IRB at National Jewish Health. All subjects gave written informed consent prior to participation in the study. A summary of study subjects' characteristics is provided in Table 1.

Skin swab bacterial cultures. Skin swabs were collected from lesional and nonlesional skin of AD patients and from skin of healthy control subjects and sent to the Clinical Microbiology lab at National Jewish Health. Blood agar plates were inoculated and cultured for 24 hours. Slide coagulase and catalase tests were performed for the identification of Staphylococcus aureus isolates.

Serum IgE assessment. Measurements of total serum IgE for AD patients were performed at Allergy and Clinical Immunology Reference Laboratory, Johns Hopkins Asthma and Allergy Center (Baltimore, Maryland, USA).

Skin tape strip collection. A total of 20 consecutive D-Squame tape strips (22 mm diameter, CuDerm) were collected from the volar surface of the forearm. Nonlesional skin from AD patients and skin from healthy control subjects were sampled. In addition, tape strip samples from lesional skin were collected from $15 \mathrm{AD}$ patients in the study group. On application of the first tape disc, 4 marks were placed around the disc with a marker so that subsequent discs could be applied to the same location. Each tape disc was placed adhesive-side up in a separate well of the two 12-well plates allocated for sample collection. Plates were kept on dry ice during the tape strip collection. Tape strips 5 and 6 were designated for lipid analysis and stored at $-80^{\circ} \mathrm{C}$ until lipids were extracted. Tape strips 11-20 were allocated for RNA extraction.

Tape strip processing for lipid extraction and protein estimation. Stratum corneum from tape strips 5 and 6 (human subjects) or tape strips 1-12 (mice) was removed by scraping tape strips in a $2 \mathrm{ml}$ water-methanol $(9: 1, \mathrm{v} / \mathrm{v})$ mixture in a Petri dish with a rubber cell scraper. Floating stratum corneum particles were carefully transferred into $8 \mathrm{ml}$ glass screw cap tubes and then subjected to a modified Bligh and Dyer extraction (34). A mixture of lipid internal standards was added during the initial step of lipid extraction to ensure absolute quantitation of targeted lipid subclasses. In brief, extraction was performed overnight by adding $0.5 \mathrm{ml}$ methanol and $1 \mathrm{ml}$ chloroform; then, phase separation was achieved by adding $1.5 \mathrm{ml}$ chloroform and $1.35 \mathrm{ml} \mathrm{2 \%}$ formic acid, by intensive vortexing, and by centrifugation $(2,000 \mathrm{~g}$ for 10 minutes). After centrifugation, the upper water-methanol phase was carefully removed. Then, the bottom chloroform phase was collected with minimal disturbance to a protein interphase and evaporated by a stream of nitrogen, and lipids were dissolved in $0.2 \mathrm{ml}$ methanol for LC-ESI-MS/MS analyses. The residual protein denaturates/pellets were dried out by a stream of nitrogen and then hydrolyzed with 1 $\mathrm{ml}$ of $2 \mathrm{~N}$ sodium hydroxide overnight at $37^{\circ} \mathrm{C}$ on a shaking water bath essentially as described in ref. 35 , with slight modifications. Tubes were periodically vortexed and sonicated on a sonicating water bath during the first hours of hydrolysis. After 16-24 hours of hydrolysis, sodium hydroxide was neutralized 
with $1 \mathrm{ml} 2 \mathrm{~N}$ hydrochloric acid, hydrolyzed proteins were centrifuged at 2,000 $\mathrm{g}$ for 5 minutes, and protein concentrations were determined using DC protein assay kit (Bio-Rad) with BSA as a protein standard. It was initially determined that BSA is not needed to be hydrolyzed for determination by DC protein assay, as both nonhydrolyzed and hydrolyzed BSA provide the same readings.

Lipid analysis by targeted lipid chromatography MS/MS. Ceramides, LPC, and SM were identified and quantified using the targeted LC-ESI-MS/MS approach on a Sciex 6500QTRAP mass spectrometer coupled with a Shimadzu Nexera X2 UHPLC system. All molecules were detected in positive ion mode. SM and LPC were detected as a transition from corresponding molecular ions to the $\mathrm{m} / \mathrm{z} 184$ (indicative of a phosphocholine group). All ceramides were detected as a transition from molecular ions to the most characteristic product ions. C18-sphingosine $N$-acylated with non-hydroxy fatty acid (NS)- and C18-sphingosine $N$-acylated with $\alpha$-hydroxy fatty acid (AS) ceramides (ceramides with C18 sphingosine as a sphingoid base) were detected as a transition to the $\mathrm{m} / \mathrm{z} 264$. Similarly, C20- and C22-NS ceramides were detected as a transition to the $\mathrm{m} / \mathrm{z} 292$ and $\mathrm{m} / \mathrm{z} 320$, correspondingly. C18-dihydrosphingosine $N$-acylated with non-hydroxy fatty acid (NDS) ceramides were detected as a transition to the $\mathrm{m} / \mathrm{z} 266$. C18-phytosphingosine $N$-acylated with nonhydroxy fatty acid (NP)- and C18-phytosphingosine $N$-acylated with $\alpha$-hydroxy fatty acid (AP) ceramides were detected as a transition to the $\mathrm{m} / \mathrm{z} 300$. EOS ceramides were detected as a transition to the $\mathrm{m} / \mathrm{z} 264$, $\mathrm{m} / \mathrm{z} 292$, and $\mathrm{m} / \mathrm{z} 320$, as our preliminary work have identified all 3 sphingoid bases being present in EOS ceramides. Global glycerolipid fatty acids were determined as fatty acid methylamides after sample hydrolysis with methylamine reagent (36). Fatty acids were determined in positive ions as a transition from corresponding fatty acid methylamide molecular ions to the $\mathrm{m} / \mathrm{z} 32$ and expressed as a relative percentage of the sum of the ions. Chromatography was performed on Ascentis Express RP-Amide $2.7 \mu \mathrm{m} 2.1 \times 50 \mathrm{~mm}$ column using gradient elution from methanol/water/formic acid (65:35:0.5, 5mM ammonium formate) to methanol/ chloroform/water/formic acid (90:10:0.5:0.5, $5 \mathrm{mM}$ ammonium formate). D7-16:0 ceramide ( $N$-16:0-D7 sphingosine), 13:0 LPC, and $N-12: 0 \mathrm{SM}$ (12:0 SM) were used as the internal standards. Standard curves of variable amounts of analites versus a fixed amount of internal standard were created for 14:0, 16:0,18:0, 20:0, 22:0, 24:1, and 24:0 analogs of ceramides, dihydroceramides, SMs, and LPCs using available standards from Avanti Polar Lipids. Relative amounts of EOS ceramides were determined in a semiquantitative way by using a correction factor from a 24:0-NS ceramide standard curve.

$R N A$ preparation from skin tape strips. Collected tape strips 11-20 from human skin were sequentially scraped into the RLT buffer (Qiagen) on the day of collection and frozen at $-80^{\circ} \mathrm{C}$. RNeasy Micro Kits (Qiagen) were used according to the manufacturer's protocol to isolate RNA from skin tape strips.

$R N A$ transcriptome gene expression and quality control. RNA Ampliseq libraries were constructed and barcoded with the Ion Ampliseq Trasncriptome Human Gene Expression Kit and methods. Barcoded RNAseq libraries were pooled and sequenced on the Ion Torrent Proton sequencer using P1 chips.

Sequencing reads were mapped to Ampliseq transcriptome target regions with the torrent mapping alignment program (TMAP) and quantified with the Ion Torrent ampliSeqRNA plug-in, using the uniquely mapping option. Duplicated sequences were removed from the FASTA file, and incorrect amplicon locations were corrected as previously reported (37). To combat potential bias from sequencing depth, the counts of each sample were downsampled to $8 \times 10^{6}$ reads. The gene counts were normalized and differential expression was performed using the DESeq2 R package (38) (distributed via Bioconductor; ref. 39).

The expression data used in this paper is not regular whole transcriptome sequencing but rather targeted amplicon sequencing. Sequence read data is generated from this amplicon library that can then be mapped and quantitated using Thermo Fisher scripts and amplicon files installed on the Ion Torrent Sequencing server. Since these scripts are not available to most readers, we provide the raw gene expression count matrix generated from the Ion Torrent server as a Supplemental Table 2 for the paper.

IL-13 Tg mice. Skin-specific inducible IL-13 Tg mice were generated in Tao Zheng's laboratory, Yale University, as described (11). The genotypes of the mice were determined by PCR using specific primers for K5-tTA and TRE-Tight-IL-13 (11). DOX was added to the drinking water $(1 \mathrm{mg} / \mathrm{ml})$ to suppress tTA and to keep the IL-13 transgene off until K5-tTA-IL-13 mice were 6 weeks old. The experiments were initiated by withdrawing DOX from the drinking water. In all experiments, $\mathrm{Tg}(-)$ littermate controls received the same amount of DOX or no DOX for the same length of time. $\operatorname{Tg}(+)$ and $\operatorname{Tg}(-)$ mice were examined for skin lesions, and clinical scores for disease severity were recorded. After mice were euthanized by carbon dioxide inhalation, lesional and nonlesional skin was excised using a $4 \mathrm{~mm}$ biopsy punch (Miltex Inc.) and preserved in TRI reagent (MilliporeSigma) for RNA extraction. Separately, 20 
skin tape strips (D-Squame tape strips, $10 \mathrm{~mm}$ diameter, $\mathrm{CuDerm}$ ) were collected from lesional and nonlesional sites of $\operatorname{Tg}(+)$ mice and from normal skin of the $\operatorname{Tg}(-)$ littermates. Prior to tape strip collection, animals were shaved, and the remaining fur was removed using Nair.

Primary human keratinocyte cell culture. Primary human keratinocyte lines were purchased from Thermo Fisher Scientific. Keratinocyte cultures were grown in EpiLife cell culture medium (Invitrogen) with human keratinocyte growth supplement S7 (Invitrogen), $0.06 \mathrm{mM} \mathrm{CaCl}_{2}$, and gentamicin/amphotericin. Keratinocytes were treated with IL-4/IL-13 (R\&D Systems) or media for 24 hours (undifferentiated), or they were differentiated with media containing $1.3 \mathrm{mM} \mathrm{CaCl}_{2}$ for up to 5 days with or without cytokines. Lipid extracts were prepared for analysis as skin tape strips above.

Quantitative PCR. Murine biopsies were preserved in TRI reagent (MilliporeSigma). Total RNA was isolated from murine skin biopsies according to manufacturer's guidelines (MilliporeSigma), followed by cleanup using Qiagen RNeasy Mini Kit (Qiagen). Qiagen RNeasy Mini Kit was used for the RNA extraction from keratinocyte cultures. RNA was reverse-transcribed into cDNA using the SuperScript VILO MasterMix (Invitrogen). RT-PCR was performed and analyzed by the dual-labeled fluorogenic probe method using ABI Prism 7300 real-time PCR machine (Applied Biosystems). Primers and probes for 18s RNA; murine TATA box binding protein (TBP); murine IL-13; murine ELOVL1-7; and human ELOVL3, ELOVL6, STAT6 were purchased from Applied Biosystems. Amplification reactions were performed in MicroAmp optical plates (Applied Biosystems) in a $25-\mu 1$ volume as previously described (20). Data was normalized to 18s RNA levels or TBP levels as endogenous controls.

siRNA transfection. Primary human keratinocytes were transfected according to the manufacturer's instructions using Lipofectamine 2000 (Invitrogen) with $20 \mathrm{nM}$ control nontargeting siRNA, STAT6 Smartpool siRNA, ELOVL3 Smartpool siRNA, and ELOVL6 Smartpool siRNA (Dharmacon) in antibiotic free media, followed by treatment with IL-4/IL-13 and differentiation for up to 5 days in $1.3 \mathrm{mM}$ $\mathrm{CaCl}_{2}$-supplemented EpiLife as above. Lipid extracts were prepared for analysis.

Cell staining and microscopy. Immunofluorescence staining was performed using keratinocytes grown on cover slips. Cells were fixed with 4\% paraformaldehyde (Fisher Scientific) and permeabilized briefly with PBS $/ 0.01 \%$ saponin/0.05\% Triton X-100 (Fisher Scientific). After blocking with SuperBlock (SkyTec), cells were incubated with anti-ELOVL3 antibody (catalog ab110872, Abcam) overnight at $4^{\circ} \mathrm{C}$ and washed 3 times with PBS/0.05\% Triton X-100, followed by incubation with FITC conjugated donkey an--rabbit F(ab')2 antibody (catalog 711096-152, Jackson ImmunoResearch Laboratories) and DAPI cell nuclei stain (MilliporeSigma) for 1 hour at room temperature. Images were taken with a Leica Microscope at 250× magnification using SlideBook 6.0 software (Intelligent Imaging Innovations).

Statistics. A 2-tailed Student $t$ test was used to determine the significance of difference between 2 groups, and 1-way ANOVA was used for comparison among multiple groups. Multiple testing correction was performed by applying Benjamini-Hochberg FDR of 0.05 for significance. All data were expressed as mean \pm SEM. Difference with $P<0.05$ was considered statistically significant.

Study approval. Human studies were approved by the IRB at National Jewish Health. All subjects provided informed consent prior to participation in the study. All procedures performed on mice were in accordance with the NIH guidelines for humane treatment of animals and were approved by the IACUC of Yale University. Mice were housed in cages with microfilters in a specific pathogen-free environment.

\section{Author contributions}

EB, EG, and DYML conceived of the study. EB and EG designed and performed experiments. IB and JJ assisted with lipid mass spectrometry experiments and lipid mass spectrometry data analysis. PT assisted with sample collection from patients and patient characterization. CFH, BNR, and KAN provided technical support with RNA extraction and real-time PCR. TZ provided IL-13 Tg mice and oversaw animal experiments, MJ performed experiments with IL-13 Tg mice. CR, ND, and MAS performed and analyzed RNA-seq experiments. EB, EG, and DYML analyzed, interpreted, and synthesized the data and wrote the manuscript.

\section{Acknowledgments}

This study was supported by NIH/NIAID grant U19 AI117673, NIH/NIAMS grant R01 AR41256, NIH/NCRR grant UL1 RR025780, and The Edelstein Family Chair of Pediatric Allergy-Immunology at National Jewish Health. 
Address correspondence to: Donald Y.M. Leung, National Jewish Health, 1400 Jackson Street, Denver, Colorado 80206, USA. Phone: 303.398.1379; Email: leungd@njhealth.org.

1. Bieber T, et al. Clinical phenotypes and endophenotypes of atopic dermatitis: Where are we, and where should we go? J Allergy Clin Immunol. 2017;139(4S):S58-S64.

2. Brunner PM, Guttman-Yassky E, Leung DY. The immunology of atopic dermatitis and its reversibility with broad-spectrum and targeted therapies. J Allergy Clin Immunol. 2017;139(4S):S65-S76.

3. Eichenfield LF, Ahluwalia J, Waldman A, Borok J, Udkoff J, Boguniewicz M. Current guidelines for the evaluation and management of atopic dermatitis: A comparison of the Joint Task Force Practice Parameter and American Academy of Dermatology guidelines. J Allergy Clin Immunol. 2017;139(4S):S49-S57.

4. Leung DY, Guttman-Yassky E. Deciphering the complexities of atopic dermatitis: shifting paradigms in treatment approaches. $J$ Allergy Clin Immunol. 2014;134(4):769-779.

5. Kihara A. Synthesis and degradation pathways, functions, and pathology of ceramides and epidermal acylceramides. Prog Lipid Res. 2016;63:50-69.

6. Janssens $\mathrm{M}$, et al. Increase in short-chain ceramides correlates with an altered lipid organization and decreased barrier function in atopic eczema patients. J Lipid Res. 2012;53(12):2755-2766.

7. Ishikawa J, et al. Changes in the ceramide profile of atopic dermatitis patients. J Invest Dermatol. 2010;130(10):2511-2514.

8. van Smeden J, et al. The importance of free fatty acid chain length for the skin barrier function in atopic eczema patients. Exp Dermatol. 2014;23(1):45-52.

9. Li S, et al. Altered composition of epidermal lipids correlates with Staphylococcus aureus colonization status in atopic dermatitis. Br J Dermatol. 2017;177(4):e125-e127.

10. Feingold KR, Elias PM. Role of lipids in the formation and maintenance of the cutaneous permeability barrier. Biochim Biophys Acta. 2014;1841(3):280-294

11. Zheng T, Oh MH, Oh SY, Schroeder JT, Glick AB, Zhu Z. Transgenic expression of interleukin-13 in the skin induces a pruritic dermatitis and skin remodeling. J Invest Dermatol. 2009;129(3):742-751.

12. Oh MH, et al. IL-13 induces skin fibrosis in atopic dermatitis by thymic stromal lymphopoietin. J Immunol. 2011;186(12):7232-7242.

13. Simpson EL, et al. Two Phase 3 Trials of Dupilumab versus Placebo in Atopic Dermatitis. N Engl J Med. 2016;375(24):2335-2348.

14. Elias PM. Lipid abnormalities and lipid-based repair strategies in atopic dermatitis. Biochim Biophys Acta. 2014;1841(3):323-330.

15. Li S, Ganguli-Indra G, Indra AK. Lipidomic analysis of epidermal lipids: a tool to predict progression of inflammatory skin disease in humans. Expert Rev Proteomics. 2016;13(5):451-456.

16. Elias PM, Wakefield JS. Mechanisms of abnormal lamellar body secretion and the dysfunctional skin barrier in patients with atopic dermatitis. J Allergy Clin Immunol. 2014;134(4):781-791.e1.

17. Sahle FF, Gebre-Mariam T, Dobner B, Wohlrab J, Neubert RH. Skin diseases associated with the depletion of stratum corneum lipids and stratum corneum lipid substitution therapy. Skin Pharmacol Physiol. 2015;28(1):42-55

18. Gruber R, et al. Filaggrin genotype in ichthyosis vulgaris predicts abnormalities in epidermal structure and function. $A m J$ Pathol. 2011;178(5):2252-2263.

19. Irvine AD, McLean WH, Leung DY. Filaggrin mutations associated with skin and allergic diseases. $N$ Engl J Med. 2011;365(14):1315-1327.

20. Howell MD, et al. Cytokine modulation of atopic dermatitis filaggrin skin expression. J Allergy Clin Immunol. 2007;120(1):150-155.

21. Cole $C$, et al. Filaggrin-stratified transcriptomic analysis of pediatric skin identifies mechanistic pathways in patients with atopic dermatitis. J Allergy Clin Immunol. 2014;134(1):82-91.

22. Lands WE. Metabolism of glycerolipides; a comparison of lecithin and triglyceride synthesis. J Biol Chem. 1958;231(2):883-888.

23. Ewald DA, et al. Meta-analysis derived atopic dermatitis (MADAD) transcriptome defines a robust AD signature highlighting the involvement of atherosclerosis and lipid metabolism pathways. BMC Med Genomics. 2015;8:60.

24. Westerberg R, et al. Role for ELOVL3 and fatty acid chain length in development of hair and skin function. J Biol Chem. 2004;279(7):5621-5629.

25. Kobayashi T, Fujimori K. Very long-chain-fatty acids enhance adipogenesis through coregulation of Elovl3 and PPAR $\gamma$ in 3T3L1 cells. Am J Physiol Endocrinol Metab. 2012;302(12):E1461-E1471.

26. Brauweiler AM, Goleva E, Leung DYM. Th2 cytokines increase Staphylococcus aureus alpha toxin-induced keratinocyte death through the signal transducer and activator of transcription 6 (STAT6). J Invest Dermatol. 2014;134(8):2114-2121.

27. Jensen JM, et al. Impaired sphingomyelinase activity and epidermal differentiation in atopic dermatitis. J Invest Dermatol. 2004;122(6):1423-1431.

28. Schmuth M, et al. Permeability barrier disorder in Niemann-Pick disease: sphingomyelin-ceramide processing required for normal barrier homeostasis. J Invest Dermatol. 2000;115(3):459-466.

29. Skolová B, et al. Ceramides in the skin lipid membranes: length matters. Langmuir. 2013;29(50):15624-15633.

30. Pullmannová $P$, et al. Permeability and microstructure of model stratum corneum lipid membranes containing ceramides with long (C16) and very long (C24) acyl chains. Biophys Chem. 2017;224:20-31.

31. Pullmannová P, Staňková K, Pospíšilová M, Skolová B, Zbytovská J, Vávrová K. Effects of sphingomyelin/ceramide ratio on the permeability and microstructure of model stratum corneum lipid membranes. Biochim Biophys Acta. 2014;1838(8):2115-2126.

32. Kim BE, Leung DY, Boguniewicz M, Howell MD. Loricrin and involucrin expression is down-regulated by Th2 cytokines through STAT-6. Clin Immunol. 2008;126(3):332-337.

33. Beck LA, et al. Dupilumab treatment in adults with moderate-to-severe atopic dermatitis. N Engl J Med. 2014;371(2):130-139.

34. Bligh EG, Dyer WJ. A rapid method of total lipid extraction and purification. Can J Biochem Physiol. 1959;37(8):911-917.

35. Dreher F, Arens A, Hostýnek JJ, Mudumba S, Ademola J, Maibach HI. Colorimetric method for quantifying human Stratum 
corneum removed by adhesive-tape stripping. Acta Derm Venereol. 1998;78(3):186-189.

36. Clarke NG, Dawson RM. Alkaline O leads to N-transacylation. A new method for the quantitative deacylation of phospholipids. Biochem J. 1981;195(1):301-306.

37. Reynolds SD, et al. Airway Progenitor Clone Formation Is Enhanced by Y-27632-Dependent Changes in the Transcriptome. Am J Respir Cell Mol Biol. 2016;55(3):323-336.

38. Love MI, Huber W, Anders S. Moderated estimation of fold change and dispersion for RNA-seq data with DESeq2. Genome Biol. 2014;15(12):550.

39. Huber W, et al. Orchestrating high-throughput genomic analysis with Bioconductor. Nat Methods. 2015;12(2):115-121. 